\title{
161. On Linear Hyperbolic System of Partial Differential Equations in the Whole Space
}

\author{
By Mitio Nagumo
}

(Comm. by K. KunugI, M.J.A., Dec. 13, 1956)

J. Leray gave the existence theorem for Cauchy problem of very general hyperbolic differential equations, but his work is not so simple to be followed easily in detail [1]. K. Friedrichs reduced the hyperbolic differential equations of second order to a symmetric system of differential equations of first order and solved the Cauchy problem in a lens-shaped domain [2]. He proved the existence of extended solutions by Hilbert space method, but showed the differentiability of solutions by somewhat complicated calculations of difference equations. P. Lax represented an elegant method which offers both the existence and the differentiability of solutions at once for the symmetric hyperbolic system of equations [3]. He reduced the problem to the case that all functions are periodic in every independent variables, but it seems me not so adequate to obtain solutions in the whole space.

The object of this paper is to give an existence theorem for Cauchy problem in the whole space and in such an abstract form that it may cover a general class of hyperbolic systems, even parabolic equations. In this note we state only the main results and related lemmas without proof. All details will be published later. Our main idea owes to Lax. Our investigation is also much stimulated by the conversations with Dr. T. Shirota and Prof. K. Yosida [4]. Especially I owe to Shirota the generalization of the operator $\Lambda$ such as (3), to make it suitable to a wide class of equations [5].

\section{Solutions without Initial Condition}

Let $x=\left(x_{1}, \cdots, x_{m}\right)$ be a variable point of $m$-dimensional Euclidean space $E^{m}$, and $u=\left(u_{1}, \cdots, u_{l}\right)$ be $l$-dimensional vector, whose components are real valued functions of $x \in E^{m}: u=u(x)$. By $(u, v)$ we denote the inner product

$$
(u, v)=\int_{E^{m}} \sum_{i=1}^{l} u_{i} v_{i} d x
$$

and $\|u\|$ denotes the norm of $u:\|u\|=V(u, u)$. With this norm we get a real Hilbert space $H_{0}$ :

$$
H_{0}=\{u ;\|u\|<\infty\} .
$$

Let $\Lambda$ be a self-adjoint linear differential operator such that 
( $\Lambda u$ is also a real $l$-dimensional vector function of $x \in E$ ),

$$
\begin{array}{cll}
(\Lambda u, v)=(u, \Lambda v) & \text { for any } & \left.u, v \in \boldsymbol{C}_{0}^{\infty}, 1\right) \\
(\Lambda u, u) \geqq(u, u) & \text { for any } & u \in \boldsymbol{C}_{0}^{\infty} .
\end{array}
$$

We define the $\Lambda$-norm of $u \in \boldsymbol{C}_{0}^{\infty}$ by

$$
\|u\|_{\Lambda}=\sqrt{(\Lambda u, u)} \quad(\geqq\|u\|) .
$$

By $H_{A}$ we denote the completion of $C_{0}^{\infty}$ with respect to the $\Lambda$-norm. Then $H_{A} \subset H_{0}$, and $H_{A}$ is a real Hilbert space with inner product $\left(u_{\Lambda} v\right)$, such that $\left(u_{\Lambda} v\right)=(\Lambda u, v)=(u, \Lambda v)$ for $u, v \in \boldsymbol{C}_{0}^{\infty}$.

By the closure $\bar{\Phi}$ of a differential operator $\Phi$ we understand that

$$
\bar{\Phi} u=v
$$

means, that there exists a sequence $u_{n} \in \boldsymbol{C}_{0}^{\infty}$ such that

$$
\left\|u_{n}-u\right\| \rightarrow 0, \quad\left\|\Phi u_{n}-v\right\| \rightarrow 0 \text {. }
$$

From now on, we understand that differential operator is always the closure of itself in ordinary sense.

We call $\Lambda$ regular with respect to $\Phi$, if for any $w \in C_{0}^{\infty}$ there exists $v \in H_{\Lambda} \frown \mathscr{D}[\Phi]^{2)}$ such that

$$
\Lambda v=w .
$$

If the coefficients of the differential operator $\Lambda$ are constants with the properties (1) and (2), then $\Lambda$ is regular with respect to similar differential operator with bounded coefficients. Elliptic differential operator whose coefficients satisfy adequate conditions is also regular to differential operator of the same order with bounded coefficients.

Theorem 1. Let $\Lambda$ satisfy the conditions (1) and (2), and be regular with respect to the linear differential operator $\Phi$. If

$$
(\Phi u, \Lambda u) \geqq(\Lambda u, u) \text { for all } u \in \boldsymbol{C}_{0}^{\infty} \text {, }
$$

then, for any $\varphi \in H_{A}$ there exists a unique $u \in H_{A}$ such that

$$
\Phi u=\varphi .
$$

To prove this we use the following idea and lemmas essentially owing to Lax. We define $\bar{\Lambda}$-norm of $v \in H_{0}$ by

$$
\|v\|_{\bar{A}}=\sup \left\{|(u, v)| /\|u\|_{A} ; 0 \neq u \in H_{A}\right\} \text {. }
$$

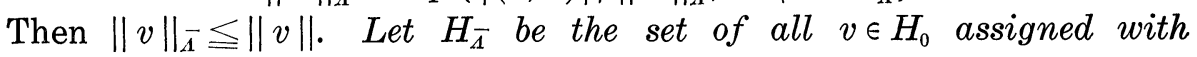
$\bar{\Lambda}$-norm.

Lemma 1. $|(u, v)| \leqq\|u\|_{\Lambda} \cdot\|v\|_{\bar{A}}$ for $u \in H_{\Lambda}, v \in H_{\bar{A}}$.

Lemma 2. Let $l(v)$ be a bounded linear functional on $H_{\bar{A}}$, then there exists a $u_{0} \in H_{\alpha}$ such that

$$
l(v)=\left(u_{0}, v\right) \quad \text { for all } \quad v \in H_{\bar{A}} .
$$

Lemma 3. If $v \in H_{\Lambda}, w \in H_{0}$ and $\Lambda v=w$, then

$$
\|w\|_{\vec{\Lambda}}=\|v\|_{\Lambda} \text {. }
$$

Lemma 4. Let $\Phi^{*}$ be the adjoint differential operator of $\Phi$, then

$$
\left\|\Phi^{*} w\right\|_{\bar{\Lambda}} \geqq\|w\|_{\bar{A}} \text {. }
$$

1) $\boldsymbol{C}_{0}^{\infty}$ means the set of $\boldsymbol{C}^{\infty}$ functions with compact carriers.

2) $\mathscr{D}[\emptyset]$ means the domain of $\boldsymbol{\emptyset}$. 


\section{Cauchy Problem}

Now we consider real $l$-dimensional vector functions of $m+1$ variables $u=u(t, x),\left(-\infty<t<\infty, x \in E^{m}\right)$. If we take for $t$ a fixed value and consider $u$ as function of $x \in E^{m}$ alone, we write it as $u=u_{(t)}$. We introduce the notation $[u, v]_{\alpha}^{\beta},(\alpha<\beta)$, by

$$
[u, v]_{\alpha}^{\beta}=\int_{\alpha}^{\beta}\left(u_{(t)}, v_{(t)}\right) d t=\int_{\alpha}^{\beta} d t \int_{E^{m}}\left(\sum_{i} u_{i} v_{i}\right) d x,
$$

and \|\|$u \|_{\alpha}^{\beta}$ by \|\|$u \|_{\alpha}^{\beta}=V \overline{[u, u]_{\alpha}^{\beta}}$.

Let $\Lambda$ be a self-adjoint linear differential operator as in $\S 1$ acting on $u_{(t)}$, i.e. $\Lambda$ contains differentiation only in $x$, but the coefficients may depend on $(t, x)$. Let us define $\left[u_{A} v\right]_{\alpha}^{\beta}$ and $\||u|\|_{\Lambda, \alpha}^{\beta}$ by

$$
\left[u_{\Lambda} v\right]_{\alpha}^{\beta}=\int_{\alpha}^{\beta}\left(u_{(t) \Lambda} v_{(t)}\right) d t=[\Lambda u, v]_{\alpha}^{\beta}=[u, \Lambda v]_{\alpha}^{\beta},
$$

and by \|\|$u \|_{\Lambda, \alpha}^{\beta}=\sqrt{\left[u_{\Lambda} u\right]_{\alpha}^{\beta}}\left(\geqq\|\| u \|_{\alpha}^{\beta}\right)$ resp.

If $\alpha=-\infty, \beta=\infty$, we write simply

$$
\left[u_{\Lambda} v\right]_{-\infty}^{\infty}=\left[u_{\Lambda} v\right], \quad\|u\|\left\|_{\Lambda, \alpha}^{\beta}=\right\|\|u\| \|_{\Lambda} .
$$

We assume, $\Lambda$ has such property that

$$
\begin{gathered}
\frac{d}{d t}(\Lambda u)=\Lambda \frac{d}{d t} u+\Lambda^{\prime} u \\
\left|\left(u, \Lambda^{\prime} u\right)\right| \leqq c^{\prime}(u, \Lambda u) \text { for all } u \in \widetilde{\boldsymbol{C}}_{0}^{\infty} .^{3)}
\end{gathered}
$$

Lemma 2.1. Let $\Lambda$ satisfy the conditions (1), (2) and (2.1), and $A$ be a differential operator acting on $u_{(t)}$ such that

$$
(A u, \Lambda u) \leqq c(u, \Lambda u) \text { for all } n \in \boldsymbol{C}_{0}^{\infty} .
$$

Then for the operator $\Phi u=\partial_{t} u-A u$, we have, if $\tau<t$,

$$
\left\|e^{-k t} u_{(t)}\right\|_{\Lambda}^{2} \leqq\left\|e^{-k \tau} u_{(\tau)}\right\|_{\Lambda}^{2}+\left(\left\|e^{-k t} \Phi u\right\|_{\Lambda, \tau}^{t}\right)^{2} \text { for all } u \in \tilde{\boldsymbol{C}}_{0}^{\infty},
$$

where $k=c+\frac{c^{\prime}}{2}$.

Lemma 2.2. Let $\Lambda$ and $A$ satisfy the same conditions as in Lemma 2.1. Then for all $u \in \widetilde{\boldsymbol{C}}_{0}^{\infty}$

$$
\left[\left(\partial_{t}-A\right) u, \Lambda u\right] \geqq-\left(c+c^{\prime} / 2\right)[u, \Lambda u] .
$$

By $\tilde{H}_{A}$ we denote the completion of $C_{0}^{\infty}$ with respect to the norm $\|u\| \|_{A}$. From Lemma 2.1, we can conclude that, if $u \in \widetilde{H}_{A}$ then $u_{(t)} \in H_{A}$ for every $t$, and $u_{(t)}$ is continuous from $(-\infty<t<\infty)$ to $H_{A}$.

Theorem 2. Let $\Lambda$ satisfy the conditions (1), (2) and (2.1) and be regular with respect to $\Phi=\partial_{t}-A$. If

$$
(A u, A u) \leqq-\left(1+c^{\prime} / 2\right)(u, \Lambda u) \text { for all } u \in C_{0}^{\infty} \text {, }
$$

then, for any $v \in \tilde{H}_{A}$, there exists a $u \in \tilde{H}_{A}$ such that

$$
\Phi u \equiv \partial_{t} u-A u=v \text {. }
$$

To prove this we use Lemma 2.2 and Theorem 1 .

3) $\widetilde{\boldsymbol{C}}_{0}^{\infty}$ means the set of all $\boldsymbol{C}_{\infty}$ functions of $(t, x)$ on $\boldsymbol{E}^{m+1}$ with compact carrier. 
Lemma 2.3. Let $\Lambda$ and $A$ satisfy the same conditions as in Theorem 2. Let $\varphi$ be such that $\varphi \in H_{A}$, and $\varphi(t, x)=0$ for $t<0$, and $u$ be the solution of

Then $u(t, x)=0$ for $t \leqq 0$.

$$
\Phi u \equiv \partial_{t} u-A u=\varphi
$$

Now let $\mathscr{F}_{A}$ be the set of functions $u(t, x)$ such that \|\|$u(t, x)\|\|_{A, 0}$ $<\infty$ for any $\tau \geqq 0$ and $u_{(t)} \in H_{A}$ is continuous from $(0 \leqq t<\infty)$ to $H_{\Lambda}$. Then we have an existence theorem for the Cauchy problem:

Theorem 3. Let $\Lambda$ satisfy the conditions (1), (2) and (2.1) and be regular with respect to $\bar{\Phi}=\partial_{t}-A$. If

$$
(A u, \Lambda u) \leqq c(u, \Lambda u) \text { for all } u \in \boldsymbol{C}_{0}^{\infty} \text {, }
$$

then, for any $v \in \mathscr{F}_{A}$ and $\varphi \in H_{A}$, there exists a unique $u \in \mathscr{F}_{A}$, such that

$$
\Phi u \equiv \partial_{t} u-A u=v, \quad u_{(0)}=\varphi \text {. }
$$

\section{References}

[1] J. Leray: Hyperbolic differential equations, Lecture in Princeton (1952).

[2] K. Friedrichs: Symmetric hyperbolic linear differential equations, Comm. Pure and Appl. Math., 6, 299-326 (1953).

[3] P. Lax: On Cauchy's problem for hyperbolic equations and the differentiability of solutions of elliptic equations, Comm. Pure and Appl. Math., 8, 615-633 (1955).

[4] K. Yosida: An operator-theoretical integration of the temporally inhomogeneous wave equation (to appear).

[5] T. Shirota: The initial value problem for linear partial differential equations with variable coefficients (to appear). 